\title{
Liver biopsy - the current view?
}

\section{S Beningfield, MB ChB, FFRad (D)}

Department of Radiology, University of Cape Town

\section{Abstract}

Liver biopsy needs careful consideration for both focal and diffuse disease, particularly in surgically-resectable lesions and with core biopsies. Increasing perceptions of the risk of needle-track seeding are added to the chance of post-procedure haemorrhage. In high-risk patients, plugged biopsy or transjugular liver biopsy may reduce the chance of adverse outcomes.

\section{Introduction}

Medical teams dealing with liver disease have been performing biopsies on the human liver for over 120 years, in the hope that the benefits of the histological (and, more recently, cytological) information derived from tissue obtained, outweigh the risk of the procedure. The biopsy is performed either for focal lesions or diffuse disease, and may produce cells for cytology, or cores of tissue for histology.

Since the introduction of the fine-needle, its comparative safety, coupled most importantly with excellent cytologists, have greatly encouraged the use of fine needle aspiration biopsies (FNABs). These are performed largely for focal lesions. There is some disagreement at exactly what level a needle is considered 'fine'; cutoffs range from an outer diameter of less than $1 \mathrm{~mm}$ (i.e. less than 19 gauge), to 22 gauge or less, using the conventional Stubs needle gauge (Fig. 1). The term 'Chiba needle', sometimes used interchangeably with fine-needle, is also confusing, being applied to needles as large as 18 gauge. At the same time improvements in the core biopsy needles and alternatives has added to the options.

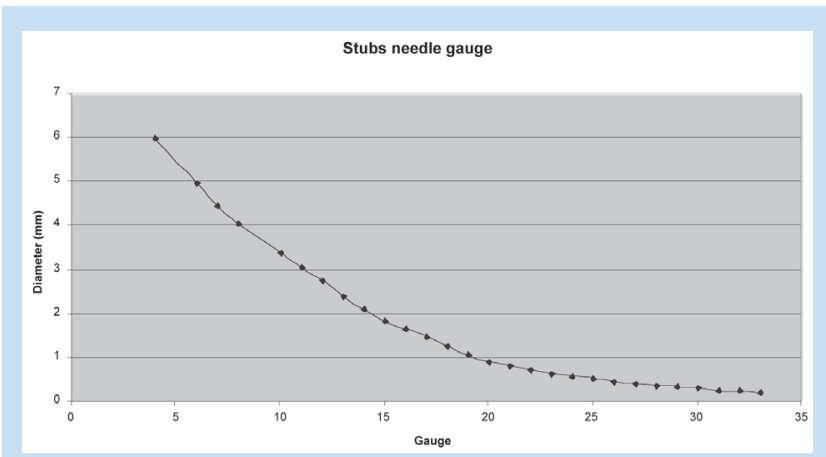

Fig. 1. Needle gauge versus outer diameter in $\mathrm{mm}$. Inner diameter ranges from $40 \%$ (smaller gauges) to $80 \%$ (larger gauges) of outer diameter.

\section{Patient preparation}

The most important points are ensuring that there is truly a need for biopsy, and that the patient's later treatment will not be compromised. For potentially resectable lesions, consultation with a liver surgeon is essential.
Clotting screens and clinical history, including known allergy (as well as to local anaesthetic) are required. In general, an International Normalised Ratio (INR) of less than 1.5 and platelets over 50 000/microl are needed for core biopsy; if not, correction of deficiencies plus possibly use of a safer technique are indicated. In these and other high-risk patients with ascites, or portal hypertension, options include plugged liver biopsy or trans-jugular liver biopsy (TJLB).

Post-procedure observation periods are subject to local policy guidelines, and usually vary from an hour for an uncomplicated FNAB, to overnight with regular observations for potentially complicated core biopsies. Sedation may be required, in which case observation after biopsy needs to be extended, and driving should be temporarily prohibited.

\section{The biopsy}

The variety of liver biopsy technique options include image-guided or non-guided, FNABs, suction or cutting core, differing needle gauges, plugged, automated, semi-automated, disposable and transjugular biopsies. The percutaneous approach is usually epigastric or intercostal, but the jugular or femoral veins and biliary drainage tracks provide alternate routes.

Cores can be obtained either by the older suction-type, e.g. Menghini needles, or the more recent cutting-core automated or semi-automated needles. These may be single units, or part of gun-type system with disposable needles.

The pathologist's requirements of the specimen depend on whether diffuse disease or focal lesions are biopsied. The ideal tissue core for diffuse disease should be $25 \mathrm{~mm}$ by $1.4 \mathrm{~mm}$, with at least 12 complete portal tracts (CPTs). ${ }^{1}$ For focal lesions, far less tissue is required.

\section{Image-guidance}

Ultrasound is most useful for both diffuse and focal disease guidance, as it provides real-time feedback on the position of the needle tip, but CT, MRI, fluoroscopy and nuclear medicine have all been used.

Focal lesions must naturally be visible ultrasonically to allow guidance. Generally either free-hand or biopsy-guide attachments are used, largely based on personal preference. Some operators find that the needle biopsy-guide interferes with the needle as corrective angulation is undertaken; others find that the guide speeds up needle tip placement. Those using the freehand technique vary in their preference for sideentry or central beam needle placment (Fig. 2).

The needle tip may stand out like a beacon, or be very difficult to see, partly depending on patient factors. Commonly used manoeuvres to improve needle tip visibility include oscillating the needle tip in rapid, low-amplitude strokes, withdrawing the stilette to allow gas to enter the needle shaft, thereby enhancing ultrasound visibility, or angling, rotating or relocating the ultrasound probe. Roughened or milled needle shafts can also assist, as can special needles with built-in tip transducers. However, there is concern that the rough shafts may encourage tumour seeding.

The aspiration for FNAB is performed while oscillating and slightly redirecting the needle tip into the target. The core biopsy needles have individual specific instructions, making sure that the firing of the needle 


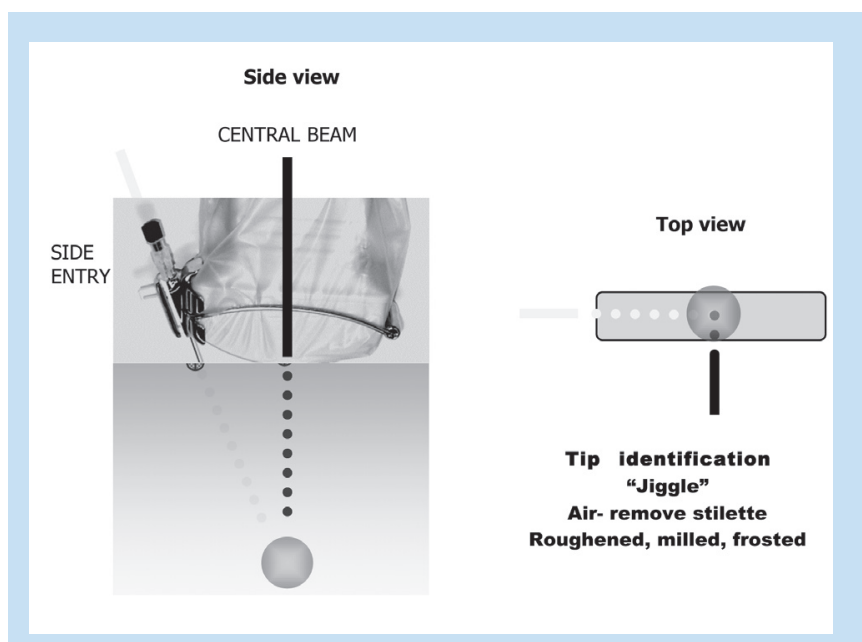

Fig. 2. Possible needle placement positions in free-hand US guidance.

delivers the specimen notch into the lesion. Newer, smaller bore cutting needles provide a crossover between FNAB and regular core biopsy needles. $^{2}$

\section{Plugged biopsy (Figs 3, 4)}

The use of a pre-placed metal sheath through which a biopsy is coaxially performed is particularly important. Commercial sets are available (Fig. 3). Plastic sheaths tends to be less controllable and more easily displaced by deep breaths or kinking.

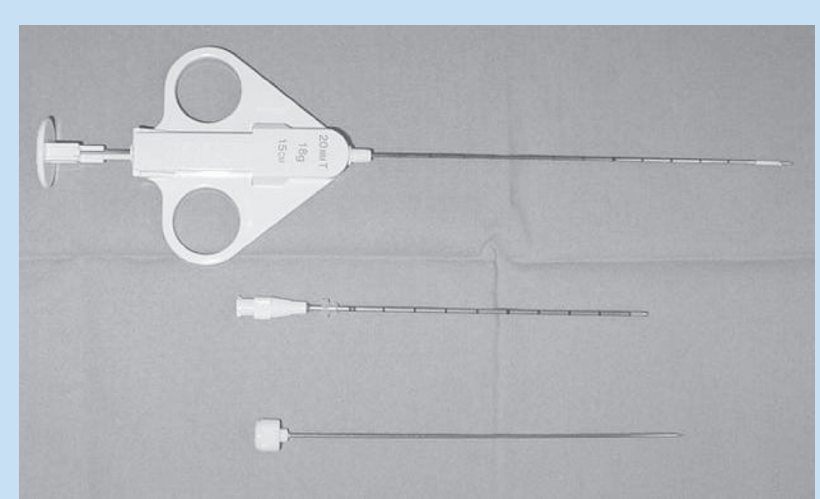

Fig. 3. Commercial plugged liver biopsy set. From top to bottom: Cutting core-biopsy needle; metal sheath; stillette.

The introduction of the gelatin sponge pledgets in the track is not always easily achieved; compressed rolled pledgets of approximately 10 $\mathrm{mm}$ by $1 \mathrm{~mm}$ are used (just wide enough to slide easily into the tip of a syringe). A test injection of pledgets prior to the biopsy is advisable.

The syringe is initially filled with saline, and a small amount of air is aspirated into the tip with the syringe tip pointing upward. This is in order to prevent premature wetting of the pledget, making it very soggy and difficult to pre-load in the syringe tip. As the metal sheath is slowly withdrawn, pledgets are successively injected by syringe into the needle. If the pledgets are too tight, re-make them in a smaller diameter. Other plugging agents have also been described, including coils or glue.

Use of contrast medium in the delivery syringe, injected under fluo- roscopy, may allow visualisation of the pledget in the needle and show the liver capsule, but pledgets should generally be placed successively as the metal sheath is withdrawn. Otherwise a potential trap arises when one incorrectly assesses the point of entry into the capsule if the liver has not been entered at the periphery. This may lead to earlier than expected exit from the liver, potentially leaving part of the track unplugged.

As hepatic veins are under low pressure, the bigger concerns are portal vein (especially with portal hypertension) and hepatic artery leaks. The likelihood of damaging the larger dangerous branches increases closer to the liver hilum, so this region should be avoided.

The intercostal artery can also be injured, despite staying close to the superior surface of the rib. This can be difficult to identify angiographically, unless selective runs of the suspect artery are undertaken.

\section{Plugged biopsy}
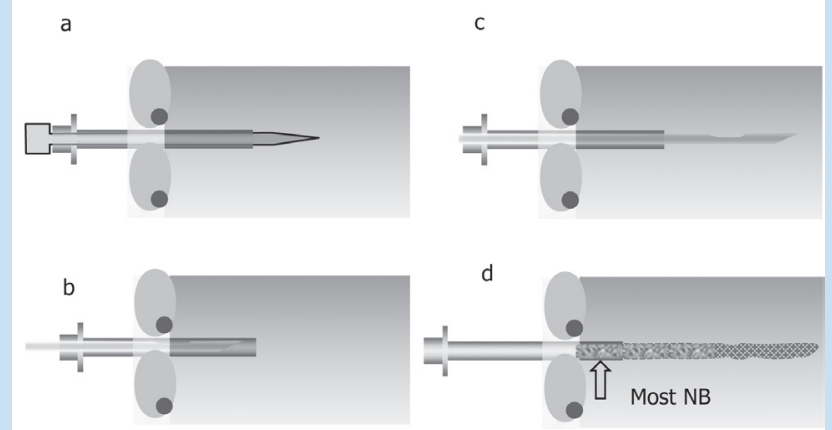

Plug with gelfoam, coils or glue

Fig. 4. Steps in performing plugged liver biopsy.

a. Metal sheath and stilette advance into liver/lesion

b. Inner stilette removed

c. Cutting core biopsy taken

d. Track plugged during stepwise withdrawal of metal sheath.

\section{TJLB (Fig. 5)}

Two subtypes of TJLB needle are available; the older modified Ross transseptal needle, relying on a suction technique, or the more modern, but more expensive, semi-automated cutting-type needle. The specimens from the latter are preferred by pathologists, although the length may not be ideal. ${ }^{3}$

In either case, right (or occasionally left) internal jugular vein access in the head-down position (to promote venous distension) allows placement of a $9 \mathrm{~F}$ Teflon transjugular catheter into the hepatic vein, usually right or middle. Passing through the right atrium is facilitated by use of a straight guidewire and a headhunter catheter, as is entry into the right hepatic vein. Thereafter either biopsy system can be used. The angle of entry into the right hepatic vein may prevent passage of the needle through the TJLB catheter, but use of gentle turning, deep breathing, advancement or traction, guidewire placement and needle bending usually permit passage.

The biopsy is usually taken in the central hepatic vein, approximately $5 \mathrm{~cm}$ from the IVC, either by a short sharp jab with the suction type needle, or by firing the cutting-needle mechanism. With the suc- 
tion needle, aspiration on withdrawal, as well as aspiration of the TJLB catheter, may produce specimens, often fragmented in cirrhosis. A check contrast injection through the catheter may occasionally show a jet of contrast leading into the peritoneum if a capsular breach has occurred. In this event, pledgets as described in the plugged biopsy technique are used to seal the leak.

An important precaution is to remove the internal jugular vein catheter (and sheath, if used) before sitting the patient upright, to avoid inadvertent air embolisation should the catheter system leak.
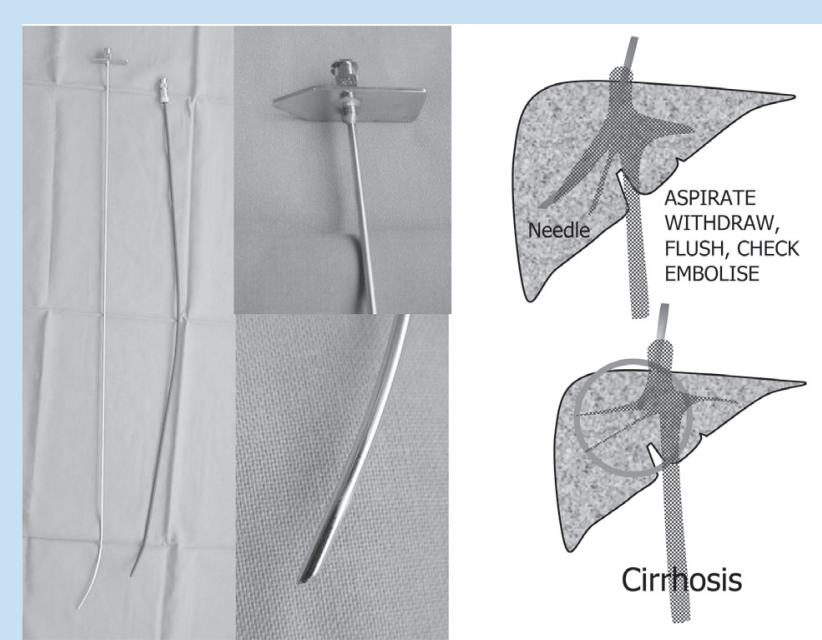

Fig. 5. Transjugular biopsy. From left to right: needle, sheath; magnified views of needle top and bottom; overview of biopsy.

\section{Liver biopsy effectiveness}

Accepting that FNAB results are highly dependent on the cytology service, excellent results are reported, especially for malignancy. Assuming the target has been sampled, the accuracy for malignancy is close to $100 \%{ }^{2}$ Core biopsies may perform somewhat better for benign lesions. ${ }^{4}$ Following a negative FNAB by a core biopsy should boost overall accuracy beyond $78 \%{ }^{2}$

Specimen adequacy can hamper the histology of diffuse disease, particularly with suction TJLB which often gives rise to fragmented specimens in cirrhosis. A comparison between plugged and TJLBs suggests better biopsy specimens with plugged biopsies, ${ }^{5}$ but possibly more bleeding.

\section{Complications}

The better understood complication is haemorrhage, with overall resultant mortality ranging between 1 in 1000 and 1 in $10000 .^{6}$ The risk also increases in patients such as children, and those with coagulopathy, ascites, and HIV (largely related to low platelet counts). ${ }^{7}$ The average mortality for core biopsy is approximately 1 in 500; mortality from FNABs for all organs is of the order of 1 in 3000 or less. $^{8}$ TJLB and plugged biopsies may carry higher risks of bleeding, ${ }^{5}$ but their main role is in high-risk patients.

The major debate currently is the risk of needle-track seeding. ${ }^{2,9,10}$ Moving from a fairly relaxed view in the early nineties, recent articles are increasingly raising concerns not only for FNABs and core biopsies, but radiofrequency ablation, percutaneous ethanol injection and drainage catheters as well. Seeding risk applies to both hepatocellular carcinoma and colonic metastases. ${ }^{2,11}$ Figures ranging from $0.6 \%$ to as high as $5.1 \%$ have been quoted, ${ }^{12}$ compared with $0.006 \%$ a decade before. ${ }^{2} \mathrm{~A}$ recent study reported a survival disadvantage in those patients with preoperative biopsy of suspected colorectal liver metastases; the biopsied group had a $32.5 \%$ 4-year survival compared to $46.7 \%$ in the unbiopsied group. ${ }^{11}$ This was, however, not a prospectively designed trial to address this specific issue, and comprised a heterogeneous group of patients. There is an argument that for focal lesions, the diagnosis is seldom altered by the biopsy. However, there may be circumstances where biopsy is vital to optimise therapy. A key component to the planning is to clear the desirability of the biopsy with the person who will be managing the patient, with this risk in mind.

Concern has been expressed that the roughened or milled surfaces of needles (with the intent of enhancing echogenicity) can drag tumour cells out along the biopsy track. Measures aimed at reducing needle track seeding include delaying needle removal, aspiration on withdrawal and radiofrequency ablation of the needle track, or using a coaxial system. ${ }^{12}$ A further critical aspect is to avoid direct puncture of a focal lesion; a cuff of normal liver must be interposed to reduce seeding risk. Anecdotal reports of 'toothpaste-like' extrusions of tumour at surgery where the tumour has been directly punctured further raise concerns for peritoneal spread.

Other major morbidity includes pain; a particularly painful biopsy should raise suspicion of a subcapsular haematoma.

\section{Conclusion}

If the information likely to be obtained from liver biopsy is balanced against the possible complications, the careful use of a variety of techniques can be helpful in further management of patients with liver problems, with a low risk of serious compromise. After confirmation with the relevant clinician, starting with a FNAB for focal lesions, reserving the core biopsy for later is advised. Tumour seeding definitely occurs, but may be overestimated.

1. Cholongitas $\mathrm{E}$, Senzolo M, Standish R, et al. A systematic review of the quality of liver biopsy specimens. Am J Clin Pathol 2006; 125:710-721.

2. Wee A. Fine needle aspiration biopsy of the liver: Algorithmic approach and current issues in the diagnosis of hepatocellular carcinoma. Cytojournal 2005; 2:7.

3. Cholongitas E, Quaglia A, Samonakis D, et al. Transjugular liver biopsy: how good it is for accurate histological interpretation? Gut 2006; 24: 2006.

4. Nyman RS, Cappelen-Smith J, Brismar J, von Sinner W, Kagevi I. Yield and complications in ultrasoundguided biopsy of abdominal lesions. Comparison of fine-needle aspiration biopsy and 1.2-mm needle core biopsy using an automated biopsy gun. Acta Radiol 1995; 36:485-490.

5. Sawyerr AM, McCormick PA, Tennyson GS, et al. A comparison of transjugular and plugged-percutaneous liver biopsy in patients with impaired coagulation. J Hepatol 1993; 17:81-85.

6. Strassburg CP, Manns MP. Approaches to liver biopsy techniques-revisited. Semin Liver Dis 2006; 26:318-327.

7. Churchill DR, Mann D, Coker RJ, et al. Fatal haemorrhage following liver biopsy in patients with HIV infection. Genitourin Med 1996; 72:62-64.

8. Smith EH. Complications of percutaneous abdominal fine-needle biopsy. Review. Radiology 1991; 178(1): 253-258.

9. Metcalfe MS, Bridgewater FH, Mullin EJ, Maddern GJ. Useless and dangerous - fine needle aspiration of hepatic colorectal metastases. BMJ 2004; 328:507-508

10. Joseph AE. Fine needle aspiration of hepatic colorectal metastases: $B M J$ enters arena of tabloid journalism. BMJ 2004; 329:290-291.

11. Jones OM, Rees M, John TG, Bygrave S, Plant G. Biopsy of resectable colorectal liver metastases causes tumour dissemination and adversely affects survival after liver resection. Br J Surg 2005; 92:1165-1168.

12. Maturen KE, Nghiem HV, Marrero JA, et al. Lack of tumor seeding of hepatocellular carcinoma after percutaneous needle biopsy using coaxial cutting needle technique. Am J Roentgenol 2006; 187:11841187. 